\title{
La traducción literaria: Nuevos retos didácticos
}

\author{
BEATRIZ M. ${ }^{a}$ RODRÍGUEZ RODRÍGUEZ \\ Universidad de Vigo \\ brodriguez@uvigo.es
}

Recibido: 18 de mayo de 2010

Aceptado: 1 de diciembre de 2010

\section{RESUMEN}

Este trabajo propone aplicar el enfoque por tareas a la didáctica de la traducción literaria para afrontar los nuevos retos didácticos que priorizan el papel que el propio alumno debe desempeñar en su propio aprendizaje. Para ello esbozaremos los aspectos más relevantes que conforman el enfoque por tareas, junto con conceptos más tradicionales que se deben integrar en este enfoque como la competencia traductora, la calidad de la traducción y la revisión y análisis crítico evaluativo de traducciones, lo que nos permitirá sugerir ejemplos de tareas que se puedan realizar en el aula. Una cuidosa planificación de los objetivos, contenidos, actividades y evaluación de cada una de las tareas permitirán que el alumno adquiera y consolide las competencias necesarias para lograr traducciones literarias de calidad.

Palabras clave: Enfoque por tareas, didáctica, traducción literaria, calidad.

\section{Literary Translation: Current Teaching Challenges}

\begin{abstract}
This paper proposes to apply task-based approach to literary translation teaching in order to face current teaching challenges, as the student's role is given special prominence. Taskbased approach will be introduced and complemented with more traditional notions such as translator's competence, translation quality, revision and contrastive assessment analysis. Then, tasks examples will be provided. A careful planning of tasks objectives, contents, activities and assessment will enable students to acquire the necessary competence to reach quality in translation.
\end{abstract}

Key words: Task-based approach, didactics, literary translation, quality.

Sumario: 1. Introducción. 2. Metodología: enfoque por tareas. 3. Competencias del traductor literario. 4. Calidad de la traducción. 5. Crítica evaluativa y revisión. Modelo de análisis de la traducción literaria. 6. Práctica de la enseñanza de la traducción literaria. 7. Conclusiones. 


\section{Introducción}

Los cambios en los planes de estudios universitarios debido a la adaptación al plan Bolonia han modificado el enfoque de la enseñanza en los estudios de traducción. Este trabajo pretende analizar los cambios en la enseñanza de la traducción literaria y los nuevos retos que se plantean. El enfoque por tareas y la utilización de las nuevas tecnologías permitirá que los alumnos controlen, bajo la supervisión del profesor, el proceso de aprendizaje y puedan adquirir y consolidar sus competencias traductológicas, sean conscientes de los factores que condicionan las decisiones de los traductores literarios, puedan analizar los problemas causados por el estilo literario del autor, conozcan las fuentes de documentación de que disponen y la situación del mercado laboral de forma que adquieran la capacidad necesaria para poderse incorporar de forma efectiva al mercado laboral.

\section{Metodología: Enfoque por tareas}

Los nuevos planes de estudios resultantes del plan Bolonia para lograr la adaptación de los planes de estudios universitarios al Espacio Europeo de Traducción han implicado, entre otros aspectos, que el enfoque didáctico pase de centrase en la figura del profesor a la figura del alumno. Los alumnos controlan su propio proceso de aprendizaje y deben adquirir las destrezas y competencias necesarias con la ayuda tutelada del profesor. Este hecho implica un cambio importante en el enfoque didáctico, cambio que también se puede apreciar en los estudios teóricos sobre metodología didáctica como veremos a continuación.

Destacan las teorías constructivistas (Reiss 2008) que se centran en el papel dado al alumnado en el proceso de aprendizaje de la traducción en base a un enfoque por proyectos que acerca a los alumnos a situaciones reales y a su contexto concreto de aplicación ${ }^{1}$. Como explica Kiraly:

rather than attempting to build up students' translation-related skills and knowledge atomistically in simulated exercises prior to translation practice, it would be much more constructive to start each pedagogical event with a highly realistic, and if possible genuine, translation project (2000: 60).

Se trata, por tanto, de anticipar las situaciones a las que los alumnos se deberán enfrentar en su futura vida laboral para que puedan adquirir las competencias necesarias que le permitan afrontar estos retos con éxito ${ }^{2}$. En una línea semejante podemos situar el enfoque transformacional mencionado por Hurtado (1999) y González

\footnotetext{
${ }^{1}$ Baker (2008: 298) afirma que el enfoque por proyectos propuesto por Vienne (1994) y Govadec (2002), entre otros, carecía de un acertado enfoque pedagógico aunque era positiva la importancia dada al mundo profesional.

${ }^{2}$ Reiss (2008) aplica el enfoque constructivista a la traducción inversa para desarrollar la competencia traductora.
} 
Davies (2004). Esta metodología también implica el desplazamiento del control del proceso de aprendizaje que pasa del profesor al alumno, un hecho que se puede aplicar directamente a los nuevos planes de estudios. González Davies aplica de forma acertada a la didáctica de la traducción el enfoque por tareas originalmente propuesto por Nunan (1989) en relación con la enseñanza de las lenguas. El planteamiento de una tarea concreta lleva consigo la realización de diferentes actividades específicas (Hurtado 1999; Willis 1996) en un contexto concreto y que permiten su consecución final centrándose en determinados objetivos y contenidos:

Concrete and brief exercises that help to practice specific points... leading along the same path towards the same end, or task, understood as a chain of activities with the same global aim and a final product. On the way, both procedural (knowhow) and declarative (know-what) knowledge are practiced and explored (Davies 2004: 22-23).

El enfoque por tareas en los estudios de traducción (Hurtado 2006) propugna un aprendizaje efectivo al estar contextualizado y relacionado con las situaciones reales del mercado laboral a las que se tendrá que enfrentar el alumno. Se trata, por tanto, de un aprendizaje específico, de un entrenamiento eminentemente práctico. Cada tarea implica la realización de diferentes actividades o micro-tareas necesarias para lograr un objetivo final y en las que se trabajan competencias de traducción concretas. Este hecho conlleva, por parte del profesor, una cuidada selección de textos y de tareas para lograr dicho objetivo. La eficacia de este enfoque se debe a la integración de todos los factores y personas que intervienen en el proceso educativo: alumnos, profesores, objetivos, contenidos, actividades, materiales, y evaluación.

A pesar de la controversia y de las divergencias entre los partidarios del enfoque por tareas y del enfoque por proyectos estamos de acuerdo con Marco (2004) y Kelly (2005), puesto que ambos intentan unir el enfoque socio-constructivista con el enfoque por tareas. En realidad en el ámbito de la didáctica de la traducción los objetivos de los planteamientos que cubren tareas o proyectos son bastante similares. Las diferencias son mínimas y ambos enfoques pretenden que el proceso de traducción sea lo más real posible en un intento de acercar el aprendizaje y el alumno al futuro mercado laboral. En todo caso, si hubiese que establecer diferencias, podríamos concluir que quizás el enfoque por tareas se pueda utilizar de forma más efectiva en las primeras actividades a desarrollar en el aula, mientras que el enfoque por proyectos se puede aplicar al final del proceso didáctico (Kelly 2005: 116).

\section{Competencias del traductor literario}

En este contexto, ¿cuáles son entonces las competencias que deben aprender nuestros alumnos? Desde nuestro punto de vista la definición de competencia del traductor propuesta por el grupo PACTE (Process of the Acquisition of Translation Competence and Evaluation) $(2000,2003,2005)$ es la más completa y coherente, ya 
que incluye todas las aptitudes y destrezas necesarias para traducir, y al mismo tiempo se basa en estudios previos de carácter empírico y dinámico. Según PACTE, la competencia traductora está formada por seis sub-tipos de competencia, que en muchos casos se solapan, y que todo buen traductor, incluido el traductor literario, debe manejar de forma eficiente. Las sub-competencias son las siguientes: competencia comunicativa en la lengua fuente y en la lengua meta (comprensión del texto fuente y expresión en el texto meta); competencia extralingüística (conocimientos acerca de la teoría de la traducción, la cultura, y el saber enciclopédico); competencia de traducción o de transferencia (capacidad que permite realizar todo el proceso de traducción, es decir, comprensión, desverbalización, re-expresión y selección del método de traducción más apropiado); competencia instrumental o profesional (capacidad de manejar las fuentes de documentación, las nuevas tecnologías, conocimiento del mercado profesional, y de las cualidades de un traductor literario); competencia psico-fisiológica (destrezas de lectura, escritura, memoria, atención, creatividad, curiosidad intelectual, rigor, perseverancia, etc.); competencia de estrategias utilizadas (todo tipo de procedimientos de los que dispone el traductor para solucionar los problemas de traducción. El traductor literario debe ser capaz de identificar y clasificar los problemas, saber cuáles son los parámetros condicionantes a la hora de tomar decisiones, corregir sus propios errores, manejar las estrategias de comprensión y formulación, y las relativas a las fuentes de documentación). PACTE (2003) afirma que el proceso de adquisición de competencia traductora es dinámico, y permite pasar de una competencia pre-traductora a una competencia profesional mediante estrategias de aprendizaje, a la vez que desarrolla y reestructura todos los tipos de sub-competencia. Una adecuada planificación de las tareas concretas a realizar permite que los alumnos adquieran y mejoren cada una de estas competencias.

\section{La calidad de la traducción}

Uno de los objetivos a tener cuenta en cualquier traducción, máxime en la traducción literaria, es la calidad de traducción. Desafortunadamente, la mayor parte de las definiciones de calidad de traducción manejadas en los Estudios de Traducción han sido demasiado genéricas, ambiguas, y subjetivas, incluso después de la publicación en 1963 por parte de E. Cary y R. W. Jumpelt del libro Quality in Translation. Proceedings of the Third Congress of the International Federation of Translators, libro que supuso en cambio significativo en el enfoque dado a este tema. Algo que también se puede decir de la obra de Christiane Schäffner Translation and Quality publicada en 1998. A partir de los años $90^{3}$ los cambios en la teoría de la traducción conllevan una cierta objetividad en los conceptos aplicados (Maier 2000: 140), evitando los conceptos genéricos y ambiguos relativos a una buena y mala traducción, y los simples listados de errores de traducción.

\footnotetext{
${ }^{3}$ En abril de 1994, por ejemplo, un congreso debatió en Nottingham, Reino Unido, el tema "Quality Assessment, Management and Control" y el estándar de calidad BS5750 (equivalente al ISO 9002) en referencia al tiempo de entrega, presentación final, relación con el cliente, facturación, etc.
} 
Resulta, por tanto, más acertado el concepto de invisibilidad que sugiere Venuti, concepto que implica que el texto meta sea transparente pero mantenga la intención del autor original:

A translated text ... is judged acceptable by most publishers, reviewers and reader when it reads fluently, when the absence of any linguistic or stylistic peculiarities makes it seem transparent, giving the appearance that it reflects the foreign writer's personality or intention or the essential meaning of the foreign text, the appearance, in other words, that the translator is not in fact a translation, but 'an original' (1995: 17).

Como mantiene Bowker (2001), no parece posible encontrar una definición de calidad de traducción que se pueda aplicar a los textos literarios debido a las particularidades que estos pueden presentar. El concepto de calidad de traducción es difícil de definir, ya que presenta "fuzzy and shifting boundaries" (Bowker 2001: 347) y se debe medir en términos comparativos (Reiss 2000: 90). Se puede concluir que los comentarios de carácter evaluativo sobre la calidad de la traducción deben tener como punto de referencia aspectos y parámetros concretos manejados en cada análisis (Chesterman 1998: 118). House explica que la evaluación y revisión debe considerar todos los aspectos y factores que condicionan el proceso de traducción:

As an evaluator one will always be forced to flexibly move from a macro-analytical focus to a micro-analytical one, from considerations of ideology, function, genre, register, to the communicative value of individual linguistic items. In taking such a multi-perspectival viewpoint, a responsible translation critic will arrive at a position where he or she can give a probabilistic reconstruction of the translator's choices, and with the support of the translator's own 'voice, ' be able to throw some light on his or her decision processes in as objective a manner as possible (2001: 256).

La aplicación de un enfoque descriptivo y funcional (Toury 1995; Nord 1997) y el análisis de los datos obtenidos permiten establecer la calidad de la traducción (Baker 2008: 207) en referencia a estos parámetros. La calidad depende también del grado de consecución del propósito o finalidad de la traducción (House 1997). Además la noción de calidad varía según la época, el lugar, las circunstancias, el género de la traducción, el propósito del autor, y el destinatario la editorial, entre otros factores. El análisis que se debe aplicar tiene por tanto cubrir todos los niveles, el análisis de la norma y del uso de la lengua junto con cuestiones socio-culturales, el contexto de la traducción y todos los elementos del proceso.

\section{Crítica evaluativa y revisión. Modelo de análisis de la traducción literaria}

Creemos que la práctica de la evaluación y crítica de textos literarios contribuye de forma efectiva a desarrollar las competencias de un traductor literario y a lograr la calidad del texto meta. Como afirma Pedersen, el análisis crítico evaluativo de tra- 
ducciones se debe basar en el análisis contrastivo del texto meta y del texto fuente (1997: 111). Siguiendo un enfoque descriptivo, comunicativo y funcional los alumnos deben realizar en primer lugar un análisis exhaustivo de los aspectos extratextuales del texto meta, del texto fuente y un estudio contrastivo de ellos en los dos textos (House 1997: 119).

Evidentemente, el análisis macro-textual es especialmente relevante en la traducción literaria (Snell-Hornby 1995). Como sugieren Lambert y van den Gorp (1985: 52-53), se analizará el tipo de encargo y de lector, la intención del autor, el género literario, el autor, la técnica narrativa, el registro, el prólogo, la división en capítulos, el contexto histórico-cultural en que se sitúan los dos textos, etc. Se analizará en detalle la intertextualidad cultural. El conocimiento cultural y enciclopédico que van adquiriendo en esta fase resulta esencial para que los estudiantes lo apliquen en la realización de traducciones posteriores. Durante las sesiones los alumnos deben analizar también la estructura de la traducción, el prólogo, y la utilización o no de notas a pie de página dependiendo del tipo de edición con lo que este hecho implica. De esta forma se practicarán los aspectos que afectan a la competencia extralingüística del alumno. Se prestará especial atención al papel de la recepción de la traducción y del autor del texto fuente en el polisistema meta (Even-Zohar 2006).

Siguiendo un análisis de "arriba a abajo" se procederá al análisis lingüístico contrastivo propiamente dicho del texto fuente y del texto meta, lo que permitirá extraer las primeras conclusiones sobre la técnica o estrategia general utilizada por el traductor como sugiere House:

Judgments of the quality of a translator depend on the large variety of factors that enter into any social evaluative assessment. Critical in the case of translation evaluation is the fact that evaluative judgments emanate from the analytic, comparative process of translation criticism, i.e., it is the linguistic analysis which provides grounds for arguing an evaluative judgment (2001: 254).

En este exhaustivo análisis textual de los dos textos, los alumnos deben identificar las unidades de análisis o segmentos, siguiendo la terminología de Toury (1995: 89). A continuación se analizarán dichos segmentos. Se identificarán los cambios o desviaciones, que Toury (1995: 57) e Leuven-Zwart (1990) denominan "shifts", y se determinarán cuales son optativos u obligatorios. Los alumnos tendrán que determinar si existe alguna justificación posible para estos cambios, o se deben a la propia inventiva del traductor o a sus criterios personales. Se analizarán las neutralizaciones, las referencias culturales, y las estrategias de extranjerización o domesticación (Venuti 1995). Dependiendo de las características específicas del texto concreto que se está analizando, se prestará especial atención a aquellos segmentos que incluyan la traducción de campos semánticos, frases hechas, refranes, juegos de palabras, y figuras retóricas, puesto que, al tratarse de textos literarios son importantes las decisiones del traductor en cuanto a los principales rasgos estilísticos y su trasvase (Boase-Beier 2006): "It is important to understand the strategy of the work as a whole, its rhythms, its imagery, its stylistic techniques; only then can we reconstruct it as a coherent whole" (Parks 2007: 248). El alumno deberá comprobar también que 
el estilo del texto fuente se ha mantenido en el texto meta, que el efecto en el lector meta es el mismo (Jin 2003: 134). El estilo del autor del texto fuente se debe respetar, pero nunca cambiar o mejorar: "the style of the original must be transferred, not improved" (Landers 2001: 90). De hecho, como explica Baker (2000: 245), el reto de mantener el estilo del autor original supone uno de los principales problemas de la traducción literaria e implica también el considerar el estilo del texto meta.

Esta evaluación de las estrategias concretas utilizadas por el traductor y los factores que la han condicionado permitirá a los alumnos mejorar su competencia en cuanto a estrategias lingüísticas, literarias y de traducción. A su vez al analizar las fuentes de documentación y las nuevas tecnologías utilizadas por el traductor, los alumnos se familiarizarán con dichos recursos, desarrollando tanto su competencia profesional como su competencia psico-fisiológica.

Durante el análisis los alumnos identificarán y clasificarán los problemas de traducción en problemas lingüísticos, culturales y pragmáticos (Nord 1996) y analizarán si las propuestas del traductor son apropiadas ya que la relevancia de cada error depende del contexto concreto en que se produce. Otra posible clasificación de problemas de traducción sería la que sugieren Martínez y Hurtado (2001: 281) y que está claramente relacionada con los tipos de sub-competencia. Los problemas serían: lingüísticos, extralingüísticos, de transferencia, psicológicos y profesionales.

Las conclusiones relativas al uso de estrategias de traducción sólo se pueden confirmar teniendo en cuenta la última parte del análisis. Es necesario aplicar parámetros o criterios de evaluación a todos los datos como único modo de lograr una evaluación objetiva (Brunette 2000: 180; Hatim \& Mason 1997: 5). Resulta imposible aplicar un único modelo de análisis a todos los textos, y lo mismo sucede con los criterios de evaluación manejados (Reiss 2000: 16), incluso aunque se trate sólo de textos literarios (Classe 2000: 1411). Cada texto tiene unas características concretas que "cannot be a priori standardized" (Gerzymisch-Arbogast 2001: 237). Por ello, tanto en esta fase como en cualquier otra del análisis evaluativo, se debe aplicar el "procedimiento helocoidal" que propone Toury (1995: 36). Según este procedimiento en la traducción de textos literarios se deben considerar en todo momento tanto los aspectos textuales como los extratextuales, debido a que el proceso siempre vuelve a fases previas. A este mismo respecto Lauscher afirma que la evaluación de la calidad de las traducciones tiene que ser un proceso suficientemente flexible como para cubrir todas las particulares de cada texto concreto que se analiza (2000: 161).

Volviendo a los criterios concretos que se deben aplicar, debemos decir que en primer lugar la forma y tipo de texto condicionan otros parámetros (Reiss 2000: 17). Aunque el enfoque debe variar ante diferentes tipos de textos literarios, otros criterios resultan más relevantes. Este es el caso de la función y el propósito del texto. Hatim y Mason (1997: 156), entre otros (House 1997: 108; Nord 1997: 166), afirman que afecta muy directamente a la de calidad de la traducción y condiciona en gran medida las decisiones del traductor. En algunas traducciones el propósito del traductor se explicita, o anticipa en el prólogo. Evidentemente, el alumno debe confirmar la veracidad y objetividad de estas afirmaciones durante el análisis. En relación con la intencionalidad del autor hay que tener en cuenta tanto el lector como el 
tipo de encargo de traducción (Schäffner 1998) y por tanto de edición, otros de los parámetros que los alumnos deben considerar a la hora de evaluar los datos que vaya obteniendo durante el análisis.

Siguiendo con la propuesta de Brunette, el contexto histórico constituye otro de los criterios centrales del análisis evaluativo porque la traducción es un proceso comunicativo y cultural que se produce en un contexto histórico concreto en un polisistema literario específico.

Non-linguistic circumstances surrounding the production of the discourse to be assessed. For assessors of general or pragmatic texts, these circumstances include the end user of the target text (in its relation to that of the source text), the position of the end user, the author (e.g. personality, experience, habits, relation to end user), the time and place in which the translation will be used, the life span of the translated text, the text type, the medium used to disseminate the text, the social situation (e.g. multilingualism) and ideological circumstances (e.g. political) surrounding the production of the target text (2000: 178-179).

A pesar de lo acertado de esta definición creemos que es demasiado amplia para aplicarla al análisis práctico y a las sesiones docentes concretas. Parece más apropiado manejar el concepto "situation" que sugieren Hatim y Mason (1997: 205) y Rabadán y Fernández (2002), y tratar a parte otros aspectos incluidos en la definición como el tipo de encargo y el lector. Los estudiantes deben ser conscientes de que los factores culturales, sociales e históricos condicionan en gran medida la práctica de la traducción.

A esto debemos añadir la importancia del criterio lógico: coherencia y cohesión. La coherencia y la cohesión de la traducción implica la estructura de la información lógica y las estrategias que conectan las partes del discurso. No se puede hablar de traducción si no se cumplen estos parámetros (Brunette 2000: 175). Los alumnos tienen que analizar los mecanismos de coherencia y cohesión utilizados por los traductores y determinar si son apropiados en el texto en cuestión.

Finalmente, se estudiará la recepción del texto en la lengua y cultura meta (Toury 1995: 56) y la posible relación del texto con otras traducciones previas (Hatim y Mason 1997: 20). La recepción complementa criterios anteriormente comentados ya que completa la función comunicativa y define la función del texto (Nord 1997: 18). Es importante, por tanto, determinar cual ha sido el papel y la posible influencia de la traducción en la cultura meta y la noción de aceptabilidad (Toury 1995: 56). Los alumnos pueden determinar si en la técnica del traductor priman las estrategias de domesticación o de extranjerización (Venuti 1995), y si existe algún tipo de justificación para ellas o se trata de una preferencia personal del traductor.

Todos estos criterios evaluativos permitirán al alumno determinar si el método de traducción y las estrategias concretas utilizadas han sido las más apropiadas en el polistema literario concreto (Even-Zohar 2006). Las conclusiones evaluativas finales relativas a la calidad de traducción deben, por tanto, tener estos parámetros como punto de referencia. Evidentemente la relevancia de cada parámetro depende del texto concreto que se esté evaluando, de ahí la imposibilidad de establecer de ante- 
mano un listado cerrado de criterios que se puedan aplicar a todos los textos. La aplicación de estos criterios evaluativos implica nuevamente el conocimiento de aspectos lingüísticos, culturales, históricos y literarios que mejorarán las competencias del alumnado.

\section{Práctica de la enseñanza de la traducción literaria}

Los conceptos esbozados en los apartados anteriores nos permiten la aplicación de un planteamiento práctico en el aula tanto si se trata de una materia concreta o de una parte de otra. El enfoque por tareas aplicado a la enseñanza de la traducción literaria les permitirá a los alumnos la simulación de la futura práctica profesional mientras continúa su proceso de aprendizaje, facilitando de esta forma el paso del aprendizaje de la revisión en sí a su posterior aplicación al ámbito profesional. Para lograr que los alumnos adquieran y mejoren las competencias de un buen traductor literario proponemos la elaboración de varias tareas. Es imprescindible también el conocimiento y uso de las TIC tanto por parte del profesor como por parte del alumno, ya que son esenciales en el futuro mercado profesional. El alumno debe conocerlas y aprender a manejarlas en aras a la efectividad y rentabilidad que su utilización implica. Debe conocer las posibilidades que ofrece Internet, la utilización de plataformas de teledocencia (por parte del alumno y del profesor: colgar los documentos de los alumnos y poder trabajar con ellos de forma efectiva, posibilidades de utilización de foros y wikis, etc.), y el manejo de programas informáticos específicos para realizar las tareas que implica la traducción de textos literarios.

En líneas generales se pueden plantear, por ejemplo, estas tareas: la revisión, evaluación y crítica de un texto literario traducido y la realización de una traducción de un texto literario.

\section{A) Revisión, evaluación y crítica de un texto literario}

Como afirma Michael Rochard (2000) la revisión es un elemento esencial en la pedagogía de la traducción puesto que relaciona el ámbito profesional con el académico, conciencia al alumno de la posible validez de varias traducciones diferentes, ayuda a que el alumno aplique los conocimientos aprendidos en diferentes disciplinas, fomenta el trabajo en equipo, y contribuye a que el profesor analice las diferentes posibilidades ofrecidas por las propuestas de los alumnos.

Para lograr al objetivo final nos debemos plantear varias micro-tareas o actividades. En primer lugar podemos trabajar el concepto de revisión. Varias clases magistrales (previo conocimiento de las diferentes corrientes teóricas sobre la traducción literaria) le aportarán al alumno conceptos básicos como el de la revisión en sí, y los diferentes tipos de revisión aplicados a textos literarios. Después de una breve introducción a los conceptos básicos, el alumno estará capacitado para realizar actividades basadas en traducciones concretas en las que identifique y analice los factores que afectan la revisión (Mossop 2001; Parra 2007) y la relación entre tra- 
ductor y revisor. También se debe trabajar con la norma europea para la prestación de servicios de traducción, la UNE-EN-15038 publicada en mayo del $2006^{4}$ y que trae consigo la consolidación del papel del revisor (Arevalillo 2005, 2006). Esta norma conlleva la obligatoriedad de que otro traductor revise el producto final logrando así una mayor calidad de la traducción.

Otra posible tarea debe tener como objetivo que los alumnos conozcan la situación del mercado editorial en España: cuáles son las principales editoriales, las asociaciones de traductores literarios, los tipos de obras literarias, publicaciones, etc. Se puede plantear como una tarea concreta en la que los alumnos en grupos pueden buscar la información bajo la supervisión del profesor. El trabajo en grupos implica un foro de discusión colectivo en el que el profesor actúa como referente y moderador.

Estas tareas previas desembocan en la aplicación del modelo de análisis crítico evaluativo previamente esbozado en el apartado 5. Evidentemente el profesor es el encargado de seleccionar de forma cuidadosa la complejidad del texto a revisar y la secuenciación concreta de la tarea. En las primeras sesiones sería interesantes manejar breves fragmentos que le permitiesen a los alumnos una primera aproximación a diferentes posibilidades: clásicos, best-seller, literatura infantil, etc. Los alumnos deben realizar el análisis de los aspectos extratextuales y textuales, prestando especial atención a la identificación de los parámetros. Será importante prestar especial atención al trasvase de los elementos que constituyen el estilo del autor original. El alumno debe ser capaz de emitir juicios críticos y objetivos sobre las estrategias utilizadas por el traductor.

\section{B) Elaboración de una traducción para una editorial}

Para lograr un efectivo enfoque por tareas esta tarea debería ser lo más real posible por lo que se podría plantear el trabajo con una editorial concreta. Se pondría plantear una novela en concreto o una colección de relatos, o incluso la traducción de teatro y poesía, siempre en base al nivel del alumnado, y objetivos y contenidos didácticos concretos.

En primer lugar los alumnos deben conocer las condiciones del encargo, las convenciones de la editorial, el lector al que va dirigida la traducción, la intención del autor, etc. Se pueden organizar grupos en los que los alumnos "actúen" como comité editorial, traductores y revisores, lo que les permitirá descubrir por sí mismos las posibles dificultades. En este momento se puede plantear una primera aproximación por parte de los alumnos a la situación del mercado laboral. Sería parte de la tarea

${ }^{4}$ La norma UN-15038 para servicios de traducción la aprobó el Comité Europeo de Normalización el 13 de abril de 2006 y se publicó en mayo de 2006. Su aplicación es obligatoria en Alemania, Austria, Bélgica, Chipre, Dinamarca, Eslovaquia, Eslovenia, España, Letonia, Finlandia, Francia, Grecia, Hungría, Irlanda, Islandia, Italia, Letonia, Lituania, Luxemburgo, Malta, Noruega, Países Bajos, Polonia, Portugal, Reino Unido, República Checa, Rumania, Suecia y Suiza. 
leer los informes del ministerio de cultura sobre los datos del mercado editorial en España, la Ley de Propiedad Intelectual, las páginas webs de las principales editoriales y asociaciones de traductores literarios y de editores. A continuación se pueden plantear el conocimiento y análisis de las fases de la traducción literaria. De forma simultánea el alumno debe conocer y saber utilizar las fuentes de la documentación literaria (Gonzalo 2005) de las que dispone por lo que pueden, por ejemplo, en grupos crear un dossier con toda la información que ellos mismos van recopilando.

Estos dos posibles ejemplos de tareas se pueden completar con actividades complementarias como por ejemplo conferencias o seminarios de traductores literarios profesionales que puedan ofrecer información práctica clave sobre su trabajo. Además, la planificación de cada tarea se debe ajustar al nivel del alumnado, combinación lingüística, materia concreta que se imparta, objetivos específicos, etc.

En cuanto a la evaluación, tanto si de trata de una materia específica de traducción literaria como si se trata de actividades concretas sobre textos literarios, ésta debe ser formativa por parte del profesor (Kelly 2005: 133) por lo que se debe considerar la realización de actividades específicas (incluidas en la planificación del profesor) en cada tarea o microtarea que le permitan al profesor explicar y comentar al alumno como avanza su aprendizaje y qué debe hacer para mejorarlo, a la vez que el profesor comprueba si el alumno está adquiriendo las competencias de traducción necesarias. Al alumno se le puede exigir y evaluar la justificación de sus decisiones y comentarios, la participación en debates, el análisis de las fuentes de documentación manejadas, etc.

\section{Conclusiones}

El enfoque por tareas aplicado a la enseñanza de la traducción de textos literarios permite que el alumno adquiera y mejore las competencias que debe reunir un traductor literario a la vez que respecta el activo papel que los alumnos tienen que desempeñar en su propia formación. Una cuidada preparación y planificación de las actividades que forman la tarea resulta esencial para cumplir los objetivos planteados. La traducción literaria es multidisciplinar e intervienen en ellas diversos factores. El profesor debe esbozar cuáles son los objetivos específicos, la destreza concreta con la que trabajar en cada caso, la competencia a mejorar, las actividades de evaluación, etc., como guía esencial para que los alumnos puedan dirigir de forma efectiva su aprendizaje del que son directamente responsables. El trabajo de los alumnos, siempre guiado por el profesor, es una preparación continua y gradual que debe llevarles a consolidar sus destrezas y habilidades como futuros traductores literarios. Los alumnos deben conocer los factores que intervienen en la traducción literaria, la situación del mercado laboral y de las asociaciones de traductores literarios, las fuentes de documentación disponibles, las nuevas tecnologías, deben ser capaces de desarrollar su espíritu crítico, e identificar el estilo del autor del texto fuente para poder trasvasarlo de forma apropiada y lograr traducciones literarias de calidad. 


\section{Referencias bibliográficas}

Arevalillo, J. J., «The EN-15308 European Quality Standard for Translation Services: What's Behind It?», The Global Insider 4 (2005). Disponible en

http://www.lisa.org/globalizationinsider/2005/04/the_en15038_eur.htm (fecha de consulta: 15 diciembre 2010).

—, «La norma europea de calidad para servicios de traducción EN-15038: por fin una realidad»,Panace@7/23 (2003), 107-111. Disponible en http:// www.medtrad.org/panacea/IndiceGeneral/n23 tribuna_Arevalillo.pdf (fecha de consulta: 15 diciembre 2010).

Baker, M. (ed.), Encyclopedia of Translation Studies. Londres y Nueva York: Routledge 2008/1998.

Boase-Beier, J., Stylistic Approaches to Translation. Manchester: St. Jerome 2006.

Bowker, L., «Towards a Methodology for a Corpus-Based Approach to Translation Evaluation», Meta 46/ 2 (2001), 345-364.

Brunette, L., «Towards a Terminology for Translation Quality Assessment. A Comparison of TQA Practices», The Translator 6/2 (2000), 169-182.

Cary, E. y Jumpelt, R.W., Quality in Translation. Proceedings of the Third Congress of the International Federation of Translation. Nueva York: Pergamon Press 1963.

Chesterman, A., Contrastive Functional Analysis. Amsterdam / Philadelphia: John Benjamins 1988.

Classe, O., Encyclopedia of Literary Translation into English. Londres: Fitzroy Dearborn Publishers 2000.

EN-15038. «European Quality Standard for Translation Services» 2006. Disponible en: http://www.cen.eu (fecha de consulta: 16 diciembre 2010).

Even-Zohar, I., «The position of Translated Literature within the Literary Polysystem», en: Weissbort, D. \& Eysteinssein, A. (eds.), Translation: Theory and Practice: A Historical Research. Oxford: Oxford University Press 2006, 429-434.

Gerzymisch-Arbogast, H., «Equivalence Parameters and Evaluation», Meta 2 (2001), 227242.

González D., Multiple Voices in the Translation Classroom. Amsterdam: John Benjamins 2004.

Gonzalo García, C. y García Yebra, V., Manual de Documentación para la traducción literaria. Madrid: Arco 2005.

Hatim, B. y Mason, I., The Translator as Communicator. Londres: Routledge 1997.

House, J., Translation Quality Assessment. A Model Revised. Gunter Narr: Tübingen 1977.

-, «Translation Quality Assessment: Linguistic Description versus Social Evaluation», Meta 46/2 (2001), 243-257.

Hurtado, A., Enseñar a traducir. Metodología en la formación de traductores e intérpretes. Madrid: Edelsa 1999.

—, «Marco teórico de la didáctica de la traducción. El enfoque por tareas de traducción», en: Gonzalo García, C. y Hernúnez P. (eds.), Corcillum. Estudios de traducción, lingüística y filología dedicados a Valentín García Yebra. Madrid: Arco 2006, 619-48.

Kelly, D., A Handbook for Translation Trainers. Manchester: St. Jerome 2005.

Kiraly, D., A Social Constructivist Approach to Translation Education. Empowerment from Theory to Practice. Manchester: St. Jerome 2000.

Kussmaul, P., Training the Translator. Amsterdam: John Benjamin 1995.

Lambert, J. y van den Gorp, H., «Describing Translations», en: Hermans, T. (ed.), The Manipulation of Literature: Studies in Literary Translation. Londres: Croom Helm 1985, 43 52. 
Landers, C. E., Literary Translation. A Practical Guide. Multilingual Matters: Universidad de Nueva Jersey 2001.

Lausher, S., «Translation Quality Assessment. Where can Theory and Practice Meet?», The Translator 6/2 (2000), 149-168.

Leuven-Zwart, K., «Translation and the Original: Similarities and Dissimilarities II», Target 2/1 (1990), 69-75.

Maier, C., «Introduction», The Translator 6/2 (2000), 137-148.

Martínez Melis, N. y Hurtado A., «Assessment in Translation Studies: Research Needs», Meta 46/2 (2001), 272-287.

Marco, J., «¿Tareas o proyectos? ¿Senderos que se bifurcan en el desarrollo de la competencia traductora?», Trans 8 (2004), 75-88.

Mossop, B., Revising and Editing for Translators. Manchester: St. Jerome 2001.

Nord, C., «El error en la traducción: categorías y evaluación», en: Hurtado Albir, A. (ed.), Enseñar a traducir. Metodología en la formación de traductores e intérpretes. Madrid: Edelsa 1996, 91-113.

—, Translation as a Purposeful Activity. Manchester: St. Jerome 1997.

Nunan, D., Designing Tasks for the Communicative Classroom. Cambridge: Cambridge University Press 1989.

PACTE, "Acquiring Translation Competence: Hypothesis and Methodological problems of a Research Project», en: Beedby, A, Ensinger D. y Presas M. (eds.), Investigating Translation. Amsterdam: John Benjamins 2000, 99-106.

—, «Building a Translation Competence Model», en: Alves, F. (ed.), Triangulating Translation: Perspectives in Process Oriented Research. Amsterdam: John Benjamins 2003.

-, «Investigating Translation Competence: Conceptual and Methodological Issues», Meta 50/2 (2005), 609-619.

Parra Galiano, S., «Propuesta metodológica para la revisión de traducciones: principios generales y parámetros», Trans 11 (2007), 197-214.

Parks, T., Translating Style. A Literary Approach to Translation. A Translation Approach to Literature. Manchester: St. Jerome 2007.

Pedersen, V. H., «Description and Criticism: Some Approaches to the English Translations of Hans Christien Andersen», en: Trósborg, A. (ed.), Text Typology and Translation. Amsterdam/ Philadelphia: John Benjamins 1997, 99-115.

Rabadán, R. y Fernández Nistal, P., La traducción inglés-español: fundamentos, herramientas y aplicaciones. ITBYTE: Universidad de León 2002.

Reiss, K., Translation Criticism: the Potentials and Limitations. Rhodes, E. F. (trad.), Möglichkeiten und Grezen der Übersetzungskritik. 1971. Münich: Max Hueber. Manchester: St. Jerome 2000.

Reiss, S., Desarrollo de la competencia traductora: teoría y práctica del aprendizaje constructivista. Comares: Granada 2008.

Rochard, M., «La revision: instrument essential de la pédagogie de la traduction», en: Collombat, O. y Gouadec, D. (eds.), Formation des traducteurs. Actes du colloque International Rennes 2 (24-25 septiembre 1999)». Paris: La Maison du Dictionnaire 2000, 77-79.

Schäffner, C. (ed.), Translation and Quality. Clevendon: Multilingual Matters 1998.

Snell-Hornby, M., «On Models and Structures and Target Text Cultures: Methods of Assessing Literary Translation», en: Marcos Bonillo, J. (ed.), La Traduction Literaria. Colleció Estudios sobre Traducció 12 Castellón: Universitat Jaume I 1995, 43-58.

Toury, G., Descriptive Translation Studies and Beyond. Amsterdam 1995.

Venuti, L., The Translator's Invisibility. Londres 1995.

Willis, J., A Framework for Task-based Learning. Londres: Longman 1996. 